\title{
Los grandes porqués de la (in)fecundidad en España
}

\author{
Albert Esteve y Rocío Treviño, Centre d'Estudis Demogràfics
}

El Instituto Nacional de Estadística acaba de publicar los microdatos de la Encuesta de Fecundidad de 2018. En un país de récords demográficos, su publicación ha llenado un vacío de más de dos décadas desde la realización de la última encuesta en 1999. La esperanza de vida en España es de las más altas del mundo y su fecundidad de las más bajas. La confluencia de ambas tendencias tiene implicaciones directas sobre la estructura de la población, debilitando su crecimiento natural y aumentando su edad media. Ante esta situación, cualquier intento de remontar la fecundidad obliga a un buen diagnóstico de las causas subyacentes. En este número de Perspectives Demogràfiques, presentamos los primeros resultados de la Encuesta de Fecundidad y exploramos las principales causas de la baja fecundidad. En particular, nos centramos en las mujeres que no han tenido hijos (infecundidad). Los resultados muestran que más de la mitad de las mujeres que no han sido madres lo hubieran querido ser y que las causas que no lo hicieron posible son diversas y varían a lo largo del ciclo de vida.

En cuestiones demográficas, España es un país de récords. Combina una de las esperanzas de vida más altas del planeta con una de las fecundidades más bajas (Castro-Martín y Martín-García, 2016). Desde los años 9os, el Índice Sintético de Fecundidad (ISF) - resumen de la fecundidad de las mujeres en edad reproductiva en un momento o año determinado - es inferior a los 1,5 hijos por mujer, situándose en 2017 en 1,3 hijos (INEbase). La baja fecundidad española va acompañada de una maternidad y paternidad muy tardías ${ }^{1}$, fruto, en buena parte, de una emancipación igualmente retardada. En España, más de la mitad de la población de 18 a 34 años en 2017 vivía con sus padres (el 61,2\%). Un dato que contrasta con el 19,2\% de los daneses o el 36,2\% de los franceses de la misma franja de edad (EU_SILC. 2017²). Los niveles coyunturales de fecundidad pueden estar reflejando un aplazamiento temporal de la maternidad debido a un periodo de crisis o cambio de patrón reproductivo, pero su prolongación en el tiempo tiene implicaciones directas sobre la descendencia final de las generaciones - el promedio de hijos que ha tenido una cohorte de mujeres u hombres. La descendencia final de las generaciones nacidas en España a mediados de los 70 s es de 1,4 hijos por mujer. Un promedio inferior al de los países nórdicos, el Reino Unido o Francia, cuya descendencia final se acerca al nivel de remplazo -2.1 por mujer- (Sobotka, 2017).

\section{LA (IN)FECUNDIDAD: ENTRE EL DESEO Y LA REALIDAD}

FIGURA 1. Distribución del número de hijos por mujer según la edad

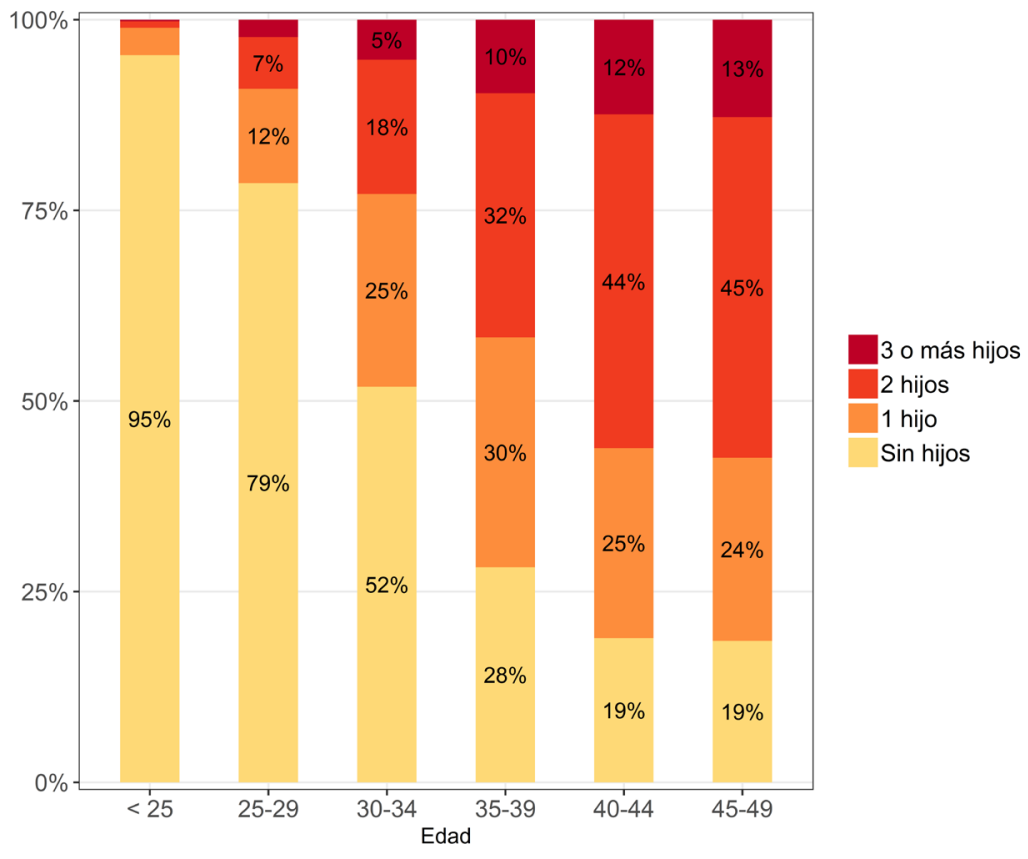

Fuente: Encuesta de Fecundidad de 2018. infecundidad de las generaciones ha crecido del $11 \%$ al 
20\% entre las mujeres nacidas en 1945 y las nacidas en 1965 y, muy probablemente, alcance el 25\% en las que lo han hecho en 1975. Una de cada cuatro mujeres de esta generación no será madre. Este nivel de infecundidad también es de récord. En un informe del Fondo de Naciones Unidas de Población, entre los 20 países del mundo seleccionados por su baja fecundidad, España destacaba, después de Japón, como el país con mayor infecundidad del mundo entre la generación de mujeres nacidas en 1974³. Esteve, Devolder y Domingo (2016) aventuraban cinco probables causas de la elevada infecundidad, pero la importancia relativa de cada una de ellas no podía establecerse debido a la falta de datos. Esto es ahora posible gracias a la publicación de la Encuesta de Fecundidad de 2018, realizada por el Instituto Nacional de Estadística (INE) sobre la base de 14.556 mujeres y 2.619 hombres. Diecinueve años después de la última Encuesta de Fecundidad (1999), el INE ha presentado esta encuesta que pregunta, entre otras cuestiones, porqué las mujeres (y los hombres) no tienen hijos. A continuación, presentamos los primeros resultados al respecto. Examinamos los valores de fecundidad, comparamos la fecundidad deseada con la observada y analizamos las razones esgrimidas por las mujeres sin hijos para no tenerlo.

La Figura 1 muestra la distribución de las mujeres por edad y número de hijos en el momento de la encuesta. Este gráfico no distingue entre las nacidas en España y las que lo hicieron en el extranjero. La fecundidad de las primeras es ligeramente inferior a la de las extranjeras (1,53 hijos por mujer versus 1,70 hijos). La proporción de mujeres sin hijos disminuye rápidamente con la edad hasta los 40 años. A partir de esta edad, se estabilizan las diferencias y tampoco varía la distribución por hijos de las mujeres que han sido madres. Más de la mitad de las mujeres entre 30 y 34 años no han sido madres. $\mathrm{Y}$ a los 40 años, sigue habiendo casi un $20 \%$ que no ha tenido ningún hijo. Por generaciones, las mujeres que tenían entre 45 y 49 años en 2018 corresponderían a las nacidas entre 1969 y 1973. De éstas, un 19\% no habría sido madre (Figura 1) y su descendencia final acabaría siendo de 1,6 hijos por mujer. En su conjunto, los datos corroboran la edad tardía al primer hijo, con un $52 \%$ de mujeres de 30 a 34 años sin hijos (un 54,9\% entre las españolas y un 38,4\% entre las extranjeras), y unos elevados niveles de infecundidad, con un 19\% mujeres sin hijos a partir de los 40.

La fecundidad observada y la deseada no suelen coincidir. En los países de baja fecundidad, la fecundidad deseada acostumbra a ser superior a la observada y estable en el tiempo. Mientras la fecundidad ha bajado, el número de hijos deseado se ha mantenido estable en las últimas décadas en Europa, en torno a los 2 hijos por mujer (Sobotka y Beaujouan, 2014). Y el porcentaje de población adulta joven que indica que, en su ideal de familia, no cabe el tener hijos, se sitúa por debajo del 5\% en los países europeos según los datos de los últimos Eurobarómetros (Miettineny Szalma, 2014).

La Figura 2 muestra la distribución por edad de las mujeres sin hijos y que no están embarazadas según el número de hijos que querrían tener. Los datos pertenecen a mujeres de distintas generaciones observadas a distintas edades. De su observación extraemos dos conclusiones importantes. La primera es que, con independencia de su edad, la mayoría de mujeres sin hijos querría o hubiera querido ser madre en algún momento de su vida. Entre las mujeres jóvenes de 25 a 29 años que no son madres (79\%), un 21\% no quiere tener hijos y más del 70\% quieren tener dos o más hijos. A edades más avanzadas, y conforme disminuye el porcentaje de mujeres sin hijos, el peso de las mujeres que no quieren tener hijos aumenta, pero su proporción sobre el conjunto de la población se mantiene constante, cerca del 20\%. La segunda conclusión es que entre las mujeres sin hijos que han superado los 40 años y, por tanto, tienen escaso recorrido para recuperar la fecundidad, una mayoría de ellas hubiera querido tenerlos. Para las mujeres de 45 a 49 años, las nacidas entre 1969 y 1973, solo un 46\% no quería tener hijos. Un $39 \%$ hubiera querido tener dos o más y un 14\%, uno solo. Si las mujeres sin hijos (19\% del total) de esa generación hubieran tenido los hijos que deseaban, su infecundidad hubiera sido del 8,7\%, menos de la mitad del porcentaje observado.

FIGURA 2. Fecundidad deseada. Mujeres que no tienen hijos ni están embarazadas

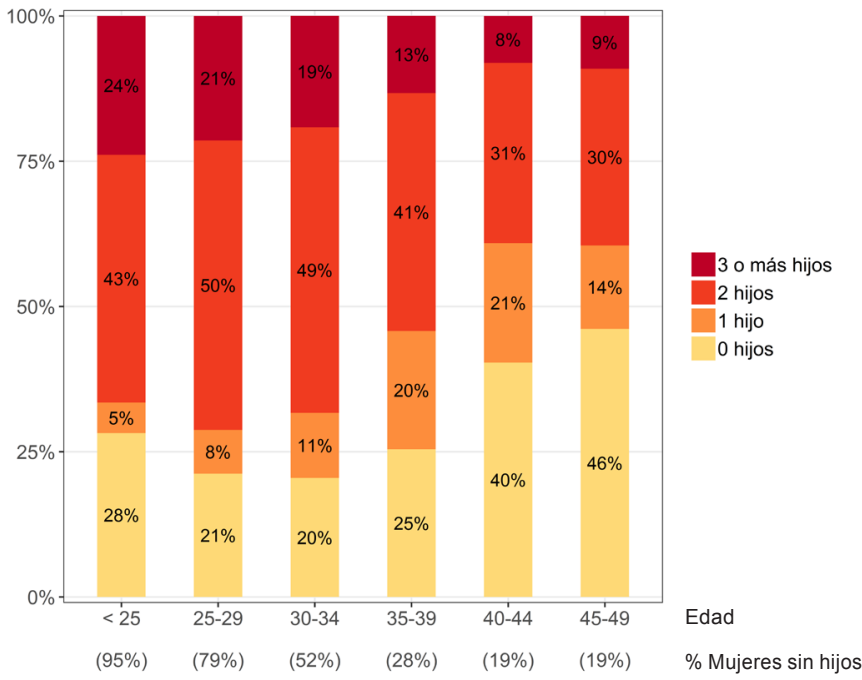

Fuente: Encuesta de Fecundidad de 2018 
¿Y cuál hubiera sido la descendencia final de esa generación de mujeres si el 54\% de mujeres que quiso ser madre, y no lo fue, hubiera tenido los hijos que deseaba? 1,8 hijos por mujer, o,2 hijos superior a la observada. Con 1,8 hijos por mujer, la descendencia final de las mujeres españolas sería superior a la de las mujeres de la misma generación nacidas en Canadá $(1,73)$, Holanda $(1,74)$ y parecida a la descendencia de las de Finlandia $(1,82)$ y solo 0,09 hijos inferior a la de las de Suecia $(1,89)^{4}$. Diversos factores, que varían y se acumulan durante el ciclo de vida de las mujeres (y hombres), impiden la materialización del potencial reproductivo deseado en España.

\section{LOS GRANDES PORQUÉS DE LA (IN)FE- CUNDIDAD}

La Encuesta de Fecundidad de 2018 preguntó a las mujeres sin hijos (y no embarazadas) el porqué no querían tener hijos. La Figura 3 muestra la distribución de los motivos para no tener hijos por edad de estas mujeres. Las respuestas se agrupan en 6 grandes grupos (Esteve, Devolder y Domingo, 2016): (i) no quiero ser madre; (ii) quiero, pero todavía soy demasiado joven o deseo seguir estudiando; (iii) problemas relacionados con la pareja; (iv) quiero, pero no reúno las condiciones económicas, laborales y de conciliación; (v) quiero, pero no me quedo embarazada o soy demasiado mayor; y (vi) otros.

Entre las mujeres menores de 25 años, cuando la mayoría de ellas no ha tenido hijos, un porcentaje muy pequeño aduce razones externas al ámbito de la decisión personal para no tenerlos, la mayoría no contemplan la maternidad porque "no toca" (74\%), ya sea porque se ven demasiado jóvenes para ser madres o porque creen incompatible la maternidad y los estudios. Un 11\% declara que no quiere ser madre y menos del 14\%, razones relacionadas con la pareja, con el mercado de trabajo o con la vivienda.

A los 25 años, las razones cambian y las circunstancias ajenas a las elecciones personales adquieren protagonismo en el $43 \%$ de las mujeres entre los 25 y 29 años, imponiéndose éstas a la mayoría de mujeres a partir de esa edad. El porcentaje de ellas que no quiere ser madre sigue estando por debajo del $12 \%$, la juventud y los estudios representan ahora el $44 \%$ de los motivos para no serlo y las razones relacionadas con la pareja (12\%) y con motivos laborales o económicos (29\%), suman un porcentaje cercano a este "ahora no toca” (por juventud o estudios). Esas categorías ganan aún más ímpetu entre los 30 y los 34 años de edad, a la par que aumenta el porcentaje de las que no quieren hijos (18\%). Las razones relaciona- das con la juventud y los estudios no desaparecen, pero su peso se reduce más de la mitad. Una de cada cinco mujeres sin hijos aduce temas relacionados con la pareja, pero son las motivaciones económicas, laborales y de conciliación las que alcanzan más importancia (36\%). El porcentaje de mujeres que no quiere tener hijos sube al $18 \%$ y aparece un $8 \%$ de mujeres que no tiene hijos por motivos de salud. A partir de los 35 años, la disminución de los porqués ligados al ámbito laboral y económico se compensa con el aumento de las mujeres que no tienen un primer hijo por motivos de salud, conjurándose, de este modo, las circunstancias que rodean la primera maternidad para vetarla. A partir de los 40 años, las razones por las que no se tienen hijos se estabilizan. La realidad y el deseo se armonizan, aumentando considerablemente el porcentaje de mujeres que no quiere ser madre y llegando al 33\% del total en el grupo de edad 45-49, triplicando así el que se registraba a los 25-29 años, cuando la elección de no ser madres empezaba a estar condicionada por las circunstancias adversas de la maternidad y no por la elección personal. A partir de los 40, el 25\% de las mujeres declara motivos de salud para no ser madre.

FIGURA 3. Razones para no tener un hijo. Mujeres sin hijos y no embarazadas

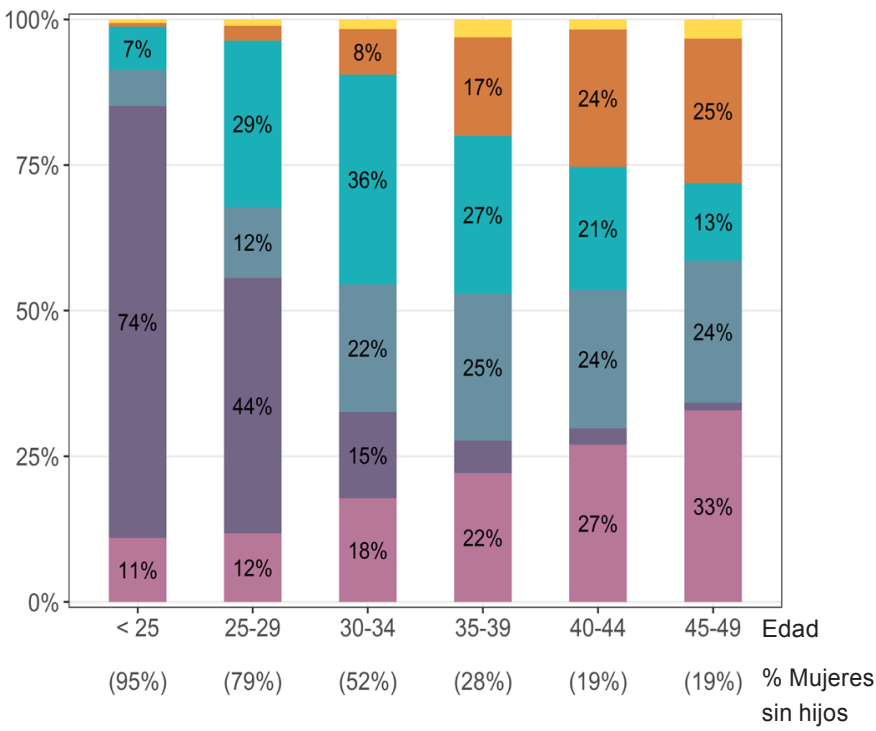

No quiero ser madre

Quiero, pero soy demasiado joven

Quiero, pero no tengo pareja

Quiero, pero no puedo por dificultades económicas, conciliación laboral-familiar

Quiero, pero no puedo por problemas de fertilidad, salud

Otras razones

Fuente: Encuesta de Fecundidad de 2018 


\section{¿HAY MARGEN PARA RECUPERAR LA FECUNDIDAD?}

Que la fecundidad en España es baja y lleva tres décadas estancada por debajo de los 1,5 hijos por mujer es algo sobradamente conocido. Sin embargo, que esta baja fecundidad es el resultado de un elevado número de mujeres y hombres que no pueden satisfacer sus deseos reproductivos por causas de diversa índole se intuía y ahora la Encuesta de Fecundidad de 2018 lo pone manifiestamente de relieve. La encuesta muestra que hay un trecho de 0,2 hijos por mujer entre la fecundidad que fue y la que pudo ser para las cohortes nacidas entre 1969 y 1973. También señala que más de la mitad de las mujeres que no fueron madres de esta generación lo quiso ser. De haberse materializado sus aspiraciones reproductivas, la descendencia final de esta generación hubiera estado por encima de los 1,8 hijos por mujer, cifra no muy lejana a la de las mujeres escandinavas.

Asimismo, la encuesta ha puesto en evidencia las causas que hay detrás de la baja fecundidad y los principales motivos del porqué las mujeres no han tenidos hijos. Los motivos son múltiples y cambiantes a lo largo de la vida reproductiva. Las razones del porqué las más jóvenes no tienen hijos se mueven en el ámbito de las decisiones personales, pero conforme se acercan y superan los treinta años de edad, afloran otras ligadas al contexto institucional y más tarde, a la salud. A partir de esta edad, las mujeres quieren mayoritariamente ser madres, pero no siempre pueden tener los hijos que desean. La transición al primer hijo es obstaculizada por motivos relacionados con la insuficiencia de recursos económicos, la situación laboral, y la dificultad de conciliar la vida laboral y familiar. El tiempo que se tarda en superar estos obstáculos obliga a posponer la decisión de ser madre hacia unas edades en las que capacidad biológica de llevar el embarazo a término es menor. Una de cada cuatro mujeres que no ha sido madre a los 40 años aduce dificultades para quedarse embarazada. Finalmente, no tener pareja o no tener la pareja adecuada es otro factor a tener en cuenta, que afecta aproximadamente a una de cada cuatro mujeres $\sin$ hijos a partir de los 35 años de edad.

A modo de conclusión, las desigualdades de oportunidades en relación con la fecundidad se juegan entre los 25 y 39 años. Las políticas públicas deberían priorizar esta franja de edad en sus actuaciones por el protagonismo que adquieren las razones de índole laboral y económico. Mejorar los niveles y condiciones de la emancipación de los jóvenes es un paso previo en esta dirección. La decisión de tener hijos no se improvisa. Es el resultado de un proceso de asentamiento personal y, la mayoría de veces, conyugal. El peso del encaje entre producción y reproducción recae sobre las biografías individuales, especialmente en las femeninas, y poco en el contexto institucional que las rodea, penalizándose así los proyectos familiares. La inercia institucional anclada en el pasado supone un freno tanto para la satisfacción de los deseos de las mujeres como para el conjunto de la sociedad. Si bien la baja fecundidad es una tendencia secular en España compartida con el resto de países desarrollados, los altos niveles de infecundidad observados en nuestro país ponen de relieve el desencuentro entre el nuevo orden de género y el marco institucional de un Estado del Bienestar puesto en cuestión.

\section{Referencias bibliográficas}

Castro-Martín, T.; Martín-García, T. (2016). "La fecundidad en España: entre las más bajas del mundo y sin muchas perspectivas de recuperación”. Panorama social (23): 11-26.

Esteve, A.; Devolder, D.; Domingo, A (2016). "La infencudidad en España: tic-tac, tic-tac, tic-tac". Perspectives Demogràfiques, 1: 1-4.

Miettinen, A.; Szalma, I. (2014) "Childlessness intentions and ideal in Europe". Finnish Yearbook of Population Research, 49: 31-55.

Sobotka, T.; Beaujouan, É. (2014). "Two Is best? The persistence of a two child family ideal in Europe". Population and Development Review, 40 (3): 391-419.
Sobotka, T. (2017). "Post-transitional fertility: childbearing postponement and the shift to low and unstable fertility levels". Vienna Institute of Demography, Working Papers, 01/2017.

Cita

Albert Esteve y Rocío Treviño (2019)

"Los grandes porqués de la (in) fecundidad en España”. Perspectives Demogràfiques, 15: 1-4.

\section{Editores}

Andreu Domingo y Albert Esteve

(Centre d'Estudis Demogràfics)

Correspondencia dirigida a

Albert Esteve

aesteve@ced.uab.cat

\section{Créditos}

Gráficos: Anna Turu

Maquetación: Xavier Ruiz Vilchez Edición: Eulàlia Camps

Enlace url

http://ced.uab.es/es/difusion/ butlleti-perspectives-demografiques

\section{Contacto}

Centre d'Estudis Demogràfics. Calle de Ca n'Altayó, Edificio E2 Universitat Autònoma de Barcelona o8193 Bellaterra / Barcelona España

Teléfono: +3493581306o E-mail: demog@ced.uab.es Web: http://ced.uab.es/es/ 